\title{
Proposição de um modelo matemático para a avaliação do desempenho de sistemas de irrigação
}

\author{
Euzebio Medrado da Silva(1), Jorge Enoch Furquim Werneck Lima(1), \\ Juscelino Antônio de Azevedo(1) ${ }^{(1)}$ Lineu Neiva Rodrigues ${ }^{(1)}$
}

\begin{abstract}
(1)Embrapa Cerrados, BR 020, Km 18, Caixa Postal 08223, CEP 73310-970 Planaltina, DF. E-mail: euzebio@cpac.embrapa.br, jorge@cpac.embrapa.br, juscelin@cpac.embrapa.br, lineu@cpac.embrapa.br
\end{abstract}

\begin{abstract}
Resumo - Modelos matemáticos têm sido utilizados para representar a distribuição dos valores de lâmina de água aplicada em uma área irrigada, informação fundamental na avaliação do desempenho de sistemas de irrigação. Apesar dos avanços, ainda não existe um modelo universalmente aceito para a descrição da distribuição dos valores de água desses sistemas. Os objetivos deste trabalho foram propor um modelo matemático para a avaliação do desempenho de sistemas de irrigação e desenvolver um fator de adequação para o cálculo da lâmina bruta a ser aplicada que agregue, em um único indicador, as medidas de uniformidade e de eficiência de aplicação de água da irrigação. Os parâmetros de ajuste do modelo proposto foram determinados por meio da rotina Solver da planilha Excel, e os indicadores de desempenho da irrigação, calculados por meio de expressões matemáticas deduzidas para uso do modelo proposto. Utilizando dados de desempenho da irrigação de um pivô-central, verificou-se que o modelo é apropriado para a análise de desempenho da irrigação e para obtenção do fator de adequação da irrigação desenvolvido, ao englobar indicadores de desempenho necessários à avaliação do sistema, simplificar os procedimentos de análise e permitir o cálculo direto da lâmina de água requerida para irrigação.
\end{abstract}

Termos para indexação: uniformidade de aplicação, eficiência de aplicação, perfil de distribuição.

\section{A mathematical model for evaluating irrigation systems performance}

\begin{abstract}
Mathematical models have been proposed to represent the applied irrigation water distribution profile, which is fundamental to evaluate the performance of irrigation systems. Even though there have been advances, so far, there is not a universally accepted model as the most adequate to fit water distribution profiles from irrigation systems. The objectives of this work were to propose a model for evaluating the performance of irrigation systems, and to develop an irrigation adjusting factor for calculating the gross water depth that takes into account both water application uniformity and efficiency measures. The fitting parameters of the proposed model were determined by using the routine Solver from the spreadsheet Excel and the water application uniformity and efficiency measures were calculated from mathematical expressions derived for this model. According to data from the evaluation of a center pivot irrigation system, the proposed model is adequate for analyzing irrigation performance and for obtaining the devised irrigation adjusting factor, by gathering the required system performance indicators. It also simplifies the analysis procedures and allows a direct calculation of the water depth demand for irrigation.
\end{abstract}

Index terms: application uniformity, application efficiency, distribution profile.

\section{Introdução}

Por melhor que seja o sistema de irrigação, a distribuição da água aplicada jamais será plenamente uniforme, e a mensuração dessa variabilidade é fundamental na avaliação do desempenho da irrigação. A variabilidade pode ser expressa na forma de um perfil decrescente de distribuição de água em que cada amostra aplicada está associada à determinada fração da área irrigada.
Segundo Hart et al. (1980), quando as perdas por escoamento superficial na área de cultivo e as perdas por condução no trajeto entre o ponto de captação e a área irrigada são desconsideradas, o desempenho da irrigação pode ser determinado por um índice de uniformidade que expresse a variabilidade espacial da água aplicada, como os coeficientes de uniformidade de Christiansen (CUC) e de distribuição (CUD), e dois índices de eficiência que reflitam o porcentual da área adequadamente 
irrigada e a eficiência alcançada na aplicação da água. Esses indicadores de desempenho podem ser obtidos diretamente dos valores de lâmina de água, medidos pontualmente, ou por meio de modelos matemáticos que, quando adequadamente ajustados, permitem a geração de funções contínuas, possibilitando a aplicação de procedimentos de cálculo de áreas e volumes, essenciais na determinação dos parâmetros de desempenho de sistemas de irrigação.

Registros teóricos de frequiência (uniforme, normal, lognormal, potencial, beta e gama) têm sido propostos para descrever a distribuição da água aplicada por diversos sistemas de irrigação (Warrick, 1983). Hart \& Reynolds (1965) empregaram a distribuição normal na avaliação de desempenho da irrigação por aspersão, enquanto Karmeli (1978) desenvolveu um modelo potencial para representar a distribuição da água infiltrada na irrigação por superfície. O modelo de distribuição estatística Beta foi reconhecido por Elliot et al. (1980) como sendo bastante flexível para descrever uma grande variedade de perfis de distribuição de água da irrigação por aspersão. Chaudry (1978) utilizou a função Gama na representação de uma variedade de perfis assimétricos de distribuição de água em sistemas de irrigação. Na irrigação por sulcos, Silva \& Hart (1992) propuseram um modelo potencial alternativo ao de Karmeli (1978) para ser aplicado nos casos em que parte da área entre sulcos permanece, efetivamente, sem receber água da irrigação. Depreende-se da variedade de propostas a inexistência de um modelo universalmente aceito que represente a distribuição de água aplicada pelos diversos sistemas de irrigação.

Os objetivos deste trabalho foram propor um modelo matemático para a avaliação de desempenho de sistemas de irrigação e desenvolver um fator de adequação para o cálculo da lâmina bruta a ser aplicada que agregue, em um único indicador, as medidas de uniformidade e de eficiência de aplicação de água.

\section{Material e Métodos}

A formulação matemática desenvolvida baseou-se no pressuposto de que as quantidades de água aplicada em sistemas de irrigação, quando ordenadas de forma decrescente, geram um perfil de distribuição que varia exponencialmente em função da área relativa acumulada, iniciando com valor máximo de lâmina, correspondente à área relativa acumulada igual a zero e finalizando com valor mínimo de lâmina, correspondente à área relativa acumulada igual à unidade. A formulação pro- posta assemelha-se ao modelo de retenção de água de Genuchten (1980), descrevendo curvas em forma de "S", e possui características da função de distribuição estatística Beta (Abramowitz \& Stegun, 1972), o que lhe confere a flexibilidade desejada no ajuste aos mais variados tipos de perfis de água aplicada.

A área relativa acumulada, associada a um valor específico de água aplicada, foi calculada por meio da relação entre a soma de todas as áreas representativas dos valores de lâmina de água aplicada maiores ou iguais ao valor especificado e a área total irrigada. Nessa associação, a área relativa acumulada pode ser interpretada como a freqüência com que se espera obter uma quantidade de água aplicada maior ou igual àquele valor especificado.

Na determinação dos parâmetros de ajuste do modelo matemático, utilizou-se a técnica de otimização da rotina Solver da planilha eletrônica Excel para minimizar a seguinte função-objetivo:

$\mathrm{SQ}=\sum_{\mathrm{i}=1}^{\mathrm{N}}\left(\mathrm{X}_{\mathrm{i}}-\hat{\mathrm{X}}_{\mathrm{i}}\right)^{2}$

em que SQ é a soma de quadrados dos erros entre os valores medidos e calculados; i é o número de ordem das quantidades de água medidas e calculadas; $\mathrm{N}$ é o número total de dados medidos; $\mathrm{X}_{\mathrm{i}}$ e $\hat{\mathrm{X}}_{\mathrm{i}}$ representam as respectivas quantidades de água medida e calculada, associadas a cada área relativa acumulada.

O modelo matemático proposto para o ajuste das quantidades de água medidas e ordenadas de forma decrescente, oriundos da avaliação de sistemas de irrigação, foi o seguinte:

$\hat{X}=X_{\text {min }}+\left(X_{\text {max }}-X_{\min }\right)\left(1-a^{n}\right)^{m}$

em que $\hat{X}$ é quantidade de água estimada pelo modelo (lâmina ou volume de água aplicada) em função da área relativa acumulada; $X_{\text {min }}$ e $X_{\max }$ são parâmetros de ajuste correspondentes à quantidade mínima e máxima da água aplicada, respectivamente; a é a área relativa acumulada, variando no intervalo de 0 a $1 ; \mathrm{me} \mathrm{n}$ são parâmetros adimensionais de ajuste.

$\mathrm{O}$ arcabouço dessa modelagem consistiu em definir a função matemática para representar o perfil de distribuição da água aplicada; derivar expressões para o cálculo dos coeficientes de uniformidade de Christiansen e de distribuição; estabelecer fórmulas para o cálculo da eficiência de aplicação de água e da área adequadamente irrigada; e desenvolver um fator de adequação para o cálculo da lâmina bruta a ser aplicada, a partir da área que se deseja irrigar adequadamente, que agregue, 
em um único indicador, as medidas de uniformidade e de eficiência de aplicação de água.

A fim de facilitar o uso do modelo proposto, todas as expressões matemáticas derivadas foram adaptadas de modo que sua solução pudesse ser obtida utilizando funções disponíveis na planilha eletrônica Excel. Existem alternativas computacionais para a implementação das soluções, entretanto, optou-se pela utilização do Excel por ser uma ferramenta amplamente difundida.

Para demonstrar a aplicabilidade do modelo proposto, empregou-se um conjunto de valores de lâmina de água medidos na avaliação de desempenho de um pivô central (Tabela 1). Nesse tipo de irrigação, o processo de coleta de água é realizado por meio de coletores dispostos radialmente e espaçados uniformemente a partir do ponto pivô, o que determina frações de áreas desiguais, representativas de cada valor coletado (Hahn \& Rosentreter, 1989). Caso fossem utilizados resultados de avaliação de desempenho de outros métodos de irrigação, a exemplo da irrigação localizada, a sistemática de organização dos dados também envolveria o relacionamento entre as quantidades de água coletada e as respectivas áreas relativas acumuladas. No entanto, neste caso, as frações de áreas representativas de cada coleta poderiam ser iguais.

\section{Resultados e Discussão}

Partindo dos dados da Tabela 1 para demonstrar a aplicação do modelo proposto (equação 2) e utilizando os procedimentos da rotina Solver na determinação dos parâmetros de ajuste do modelo, obtiveram-se os seguintes resultados: $X_{\min }=10,6 \mathrm{~mm} ; X_{\max }=16,5 \mathrm{~mm}$; $\mathrm{n}=0,7615 ; \mathrm{m}=1,1283$, com uma soma de quadrados de erros $S Q_{\text {erro }}=2,4285$. Nessa otimização, os parâmetros de ajuste foram submetidos às seguintes restrições: $X_{\max } \leq$ maior valor de $X_{i} ; X_{\min } \geq 0 ; m \geq 0,0001 \mathrm{e}$ $\mathrm{n} \geq 0,0001$. Na Figura 1, apresenta-se o gráfico do perfil de distribuição de água ajustada aos valores pontuais observados. Na implementação dessa modelagem, a lâmina média indicada foi considerada, somente para fins de análise, igual ao requerimento de água da cultura.

A fórmula para o cálculo da média geral $\overline{\mathrm{X}}$ foi derivada da integração da variável $\hat{X}$, definida pela equação 2 , no intervalo de 0 a 1 , resultando em:

$$
\bar{X}=X_{\text {min }}+\left(X_{\text {max }}-X_{\text {min }}\right) \int_{0}^{1}\left(1-a^{n}\right)^{m} d a
$$

Com a resolução da integral da equação 3, aplicando a definição da função Beta (Abramowitz \& Stegun, 1972), obteve-se a seguinte equação:

$\int_{0}^{1}\left(1-\mathrm{a}^{\mathrm{n}}\right)^{\mathrm{m}} \mathrm{da}=(1 / \mathrm{n}) \mathrm{B}(1 / \mathrm{n} ; \mathrm{m}+1)$.

Como o cálculo da função Beta, representada por $\mathrm{B}(1 / \mathrm{n} ; \mathrm{m}+1)$, não está diretamente disponível na planilha Excel, foi necessário converter a equação 4 de forma que esta fosse expressa em termos da função Gama. Essa substituição foi feita utilizando a relação existente entre as funções Beta e Gama, apresentada em Abramowitz \& Stegun (1972). Assim, a equação 4 foi transformada em:

$\int_{0}^{1}\left(1-\mathrm{a}^{\mathrm{n}}\right)^{\mathrm{m}} \mathrm{da}=\frac{\mathrm{m}}{(1+\mathrm{mn})} \frac{\Gamma(1 / \mathrm{n}) \Gamma(\mathrm{m})}{\Gamma(1 / \mathrm{n}+\mathrm{m})}$.

Ao serem considerados os parâmetros de ajuste do modelo obtidos com os dados da Tabela 1, o resultado da integral foi igual a 0,40034 .

Inserindo a equação 5 na 3 , o valor médio de $X$ pode ser calculado da seguinte forma:

$\bar{X}=X_{\text {min }}+\left(X_{\text {max }}-X_{\text {min }}\right) \frac{m}{(1+m n)} \frac{\Gamma(1 / n) \Gamma(m)}{\Gamma(1 / n+m)}$.

Os valores da função Gama $\Gamma$ ( ) podem ser encontrados em tabelas ou expressões algébricas (Abramowitz $\&$ Stegun, 1972), bem como por meio de funções disponíveis na planilha eletrônica Excel, em que para o caso do parâmetro $\mathrm{m}$, o valor correspondente à sua função Gama pode ser calculado como:

$\Gamma(\mathrm{m})=\operatorname{EXP}(\operatorname{LNGAMA}(\mathrm{m}))$, em que $\operatorname{EXP}()$ e LNGAMA() são funções do programa Excel. Os demais parâmetros da equação $6\left(X_{\min }, X_{\max }, m\right.$ e n) são determinados diretamente pelo processo iterativo de otimização. Dessa forma, aplicando os valores dos parâmetros de ajuste encontrados, relativos aos dados da Tabela 1 , na equação 6 , obteve-se a lâmina média $\overline{\mathrm{X}}=12,96 \mathrm{~mm}$, enquanto a média ponderada calculada diretamente com os dados medidos (Tabela 1) foi de $12,92 \mathrm{~mm}$, denotando, nesse caso, elevado grau de concordância entre os valores medidos e calculados.

A transição entre as áreas adequadamente irrigada e deficientemente irrigada foi definida por $a_{L}$ que determina o ponto de interseção entre o descrito pelo modelo e a lâmina média aplicada. Esse valor pode ser obtido 
por meio da substituição de $\hat{\mathrm{X}}$ (equação 1) por $\overline{\mathrm{X}}$, resultando na seguinte relação:

$\mathrm{a}_{\mathrm{L}}=\left\{1-\left[\left(\overline{\mathrm{X}}-\mathrm{X}_{\min }\right) /\left(\mathrm{X}_{\max }-\mathrm{X}_{\min }\right)\right]^{1 / \mathrm{m}}\right\}^{1 / \mathrm{n}}$.

No caso exemplificado na Tabela 1 e Figura 1, o valor de $a_{L}$ encontrado foi de 0,4627 . Isto significa que $46,27 \%$ da área total do pivô central avaliado foi adequadamente irrigada.

Para o desenvolvimento da fórmula de cálculo do coeficiente de uniformidade de Chistiansen (CUC) a partir do modelo proposto (Equação 2), considerou-se a seguinte definição:

$\mathrm{CUC}=100(1-|\overline{\mathrm{D}}| / \overline{\mathrm{X}})$

em que $|\overline{\mathrm{D}}|$ é a média dos desvios absolutos dos volumes ou lâminas de água aplicada em relação à média geral $\overline{\mathrm{X}}$.

No cálculo da média absoluta dos desvios $|\overline{\mathrm{D}}|$, utilizou-se a seguinte definição (Warrick, 1983):
$|\bar{D}|=\int_{0}^{a_{L}}(\hat{X}-\bar{X}) d a+\int_{a_{L}}^{1}(\bar{X}-\hat{X}) d a$

Simplificando a equação 9, tem-se que:

$|\overline{\mathrm{D}}|=2\left[\int_{0}^{\mathrm{a}_{\mathrm{t}}} \hat{\mathrm{X}} \mathrm{da}-\overline{\mathrm{X}} \mathrm{a}_{\mathrm{L}}\right]$.

Com a substituição do termo $\hat{\mathrm{X}}$ pela definição dada na equação 2 e realizando-se as simplificações possíveis, deduziu-se a seguinte expressão:

$|\overline{\mathrm{D}}|=2\left(\mathrm{X}_{\text {max }}-\mathrm{X}_{\text {min }}\right)\left[\int_{0}^{\mathrm{a}_{\mathrm{L}}}\left(1-\mathrm{a}^{\mathrm{n}}\right)^{\mathrm{m}} \mathrm{da}-\left(\overline{\mathrm{X}}-\mathrm{X}_{\text {min }}\right) \mathrm{a}_{\mathrm{L}}\right]$

Resolvendo a integral da equação 11 e aplicando-se a definição da função Beta incompleta (Abramowitz \& Stegun, 1972), obtém-se a equação 12:

$\int_{0}^{a_{L}}\left(1-a^{n}\right)^{m} d a=(1 / n) B_{a_{L}^{n}}(1 / n ; m+1)$

Tabela 1. Valores de lâmina de água coletada, ordenados de forma decrescente na modelagem da distribuição da água aplicada por um pivô central.

\begin{tabular}{|c|c|c|c|c|c|c|c|}
\hline $\begin{array}{l}\text { Ordem } \\
\text { (i) }\end{array}$ & $\begin{array}{r}\text { Posição } \\
\left(\mathrm{d}_{\mathrm{i}}, \mathrm{m}\right)\end{array}$ & $\begin{array}{c}\text { Área relativa } \\
\text { acumulada }^{(1)}\left(\mathrm{a}_{\mathrm{k}}\right)\end{array}$ & $\begin{array}{l}\text { Lâmina } \\
\text { (mm) }\end{array}$ & $\begin{array}{l}\text { Ordem } \\
\text { (i) }\end{array}$ & $\begin{array}{c}\text { Posição } \\
\left(\mathrm{d}_{\mathrm{i}}, \mathrm{m}\right)\end{array}$ & $\begin{array}{c}\text { Área relativa } \\
\text { acumulada }^{(1)}\left(a_{\mathrm{k}}\right)\end{array}$ & Lâmina (mm) \\
\hline 1 & 82 & 0,018 & 16,5 & 23 & 174 & 0,528 & 12,5 \\
\hline 2 & 70 & 0,033 & 16,0 & 24 & 186 & 0,569 & 12,5 \\
\hline 3 & 78 & 0,050 & 16,0 & 25 & 18 & 0,573 & 12,0 \\
\hline 4 & 58 & 0,063 & 15,6 & 26 & 38 & 0,581 & 12,0 \\
\hline 5 & 74 & 0,079 & 15,5 & 27 & 42 & 0,590 & 12,0 \\
\hline 6 & 122 & 0,106 & 15,5 & 28 & 90 & 0,610 & 12,0 \\
\hline 7 & 46 & 0,116 & 15,0 & 29 & 94 & 0,631 & 12,0 \\
\hline 8 & 50 & 0,127 & 15,0 & 30 & 162 & 0,666 & 12,0 \\
\hline 9 & 62 & 0,140 & 15,0 & 31 & 170 & 0,703 & 12,0 \\
\hline 10 & 86 & 0,159 & 15,0 & 32 & 98 & 0,725 & 11,5 \\
\hline 11 & 126 & 0,187 & 15,0 & 33 & 106 & 0,748 & 11,5 \\
\hline 12 & 134 & 0,216 & 15,0 & 34 & 178 & 0,787 & 11,5 \\
\hline 13 & 66 & 0,230 & 14,4 & 35 & 190 & 0,828 & 11,5 \\
\hline 14 & 138 & 0,260 & 14,0 & 36 & 30 & 0,835 & 11,4 \\
\hline 15 & 54 & 0,272 & 13,5 & 37 & 34 & 0,842 & 11,4 \\
\hline 16 & 118 & 0,298 & 13,5 & 38 & 102 & 0,865 & 11,0 \\
\hline 17 & 130 & 0,326 & 13,5 & 39 & 110 & 0,889 & 11,0 \\
\hline 18 & 146 & 0,358 & 13,5 & 40 & 182 & 0,928 & 11,0 \\
\hline 19 & 150 & 0,391 & 13,5 & 41 & 22 & 0,933 & 10,5 \\
\hline 20 & 154 & 0,425 & 13,5 & 42 & 26 & 0,939 & 10,5 \\
\hline 21 & 142 & 0,456 & 13,2 & 43 & 114 & 0,964 & 10,5 \\
\hline 22 & 158 & 0,490 & 13,0 & 44 & 166 & 1,000 & 10,5 \\
\hline
\end{tabular}

Pesq. agropec. bras., Brasília, v.39, n.8, p.741-748, ago. 2004 
em que o termo $a_{L}^{n}$ representa o limite superior da função Beta incompleta e $(1 / \mathrm{n})$ e $(m+1)$, os parâmetros para sua avaliação.

Segundo Abramowitz \& Stegun (1972), as funções Beta incompleta e completa se relacionam da seguinte forma:

$\mathrm{B}_{\mathrm{a}_{\mathrm{L}}^{\mathrm{n}}}(1 / \mathrm{n} ; \mathrm{m}+1)=\mathrm{I}_{\mathrm{a}_{\mathrm{L}}^{\mathrm{n}}}(1 / \mathrm{n} ; \mathrm{m}+1) \mathrm{B}(1 / \mathrm{n} ; \mathrm{m}+1)$.

Considerando as igualdades estabelecidas pelas equações 4 e 5, deduziu-se a seguinte equivalência:

$\mathrm{B}(1 / \mathrm{n} ; \mathrm{m}+1)=\frac{\mathrm{mn}}{(1+\mathrm{mn})} \frac{\Gamma(1 / \mathrm{n}) \Gamma(\mathrm{m})}{\Gamma(1 / \mathrm{n}+\mathrm{m})}$

Segundo Abramowitz \& Stegun (1972), a função $I_{a_{L}^{n}}(1 / n ; m+1)$ se relaciona com a função de distribuição $\mathrm{F}$ que se encontra disponível no Excel. Essa igualdade pode ser expressa da seguinte maneira:

$\mathrm{I}_{\mathrm{a}_{\mathrm{L}}^{\mathrm{n}}}(1 / \mathrm{n} ; \mathrm{m}+1)=\operatorname{DISTF}\left[\left(\mathrm{a}_{\mathrm{L}}^{-\mathrm{n}}-1\right) /(\mathrm{nm}+\mathrm{n}) ; 2(\mathrm{~m}+1) ; 2(1 / \mathrm{n})\right](15)$ em que a expressão DISTF[ ] é a função estatística da planilha eletrônica Excel que retorna o valor da distribuição $\mathrm{F}$ avaliada em $\left[\left(\mathrm{a}_{\mathrm{L}}^{-\mathrm{n}}-1\right) /(\mathrm{nm}+\mathrm{n})\right]$, com $2(\mathrm{~m}+1)$ graus de liberdade no numerador e $2(1 / \mathrm{n})$ graus de liberdade no denominador.

Ao serem introduzidas as equações 14 e 15 na equação 13 e esta na equação 12, deduziu-se o seguinte:

$\int_{0}^{a_{L}}\left(1-a^{n}\right)^{m} d a=\left\{\operatorname{DISTF}\left[\left(a_{L}^{-n}-1\right) /(n m+n) ;\right.\right.$
$\left.2(m+1) ; 2(1 / n)]\left[\frac{m}{(1+m n)} \frac{\Gamma(1 / n) \Gamma(m)}{\Gamma(1 / n+m)}\right]\right\}$.

Substituindo-se a equação 16 na equação 12 , obtevese a seguinte expressão:

$$
\begin{aligned}
& |\overline{\mathrm{D}}|=2\left(\mathrm{X}_{\text {max }}-\mathrm{X}_{\text {min }}\right)\left\{\operatorname { D I S T F } \left[\frac{\mathrm{a}_{\mathrm{L}}^{-\mathrm{n}}-1}{\mathrm{n}(\mathrm{m}+1)} ; 2(\mathrm{~m}+1) ;\right.\right. \\
& \left.2(1 / \mathrm{n})]\left[\frac{\mathrm{m}}{(1+\mathrm{mn})} \frac{\mathrm{I}(1 / \mathrm{n}) \Gamma(\mathrm{m})}{\mathrm{I}(1 / \mathrm{n}+\mathrm{m})}\right]-\left(1-\mathrm{a}_{\mathrm{L}}^{\mathrm{n}}\right)^{\mathrm{m}} \mathrm{a}_{\mathrm{L}}\right\}
\end{aligned}
$$

Considerando os parâmetros do modelo proposto, calculados com os dados da Tabela 1, obteve-se, da planilha "Excel", o valor de DISTF[0,4926; 4,2566; $2,6264]=0,7537$. Empregando os demais resultados já conhecidos na equação 17 , obteve-se $|\overline{\mathrm{D}}|=1,38 \mathrm{~mm}$. Com os valores de $|\overline{\mathrm{D}}|$ e $\overline{\mathrm{X}}$ já determinados, utilizando a equação 8 , calculou-se o CUC $=89,35 \%$. O valor de CUC, calculado diretamente dos dados medidos (Tabela 1 ), foi de $89,17 \%$, valor muito próximo do calculado empregando o modelo proposto.
$\mathrm{Na}$ derivação da fórmula de cálculo do coeficiente de uniformidade de distribuiçãa (CUD) baseado no modelo proposto (equação 2), utilizou-se a seguinte definição:

$\operatorname{CUD}=100\left(\overline{\mathrm{X}}_{\mathrm{q}} / \overline{\mathrm{X}}\right)$

em que $\bar{X}_{q}$ é a média do quartil inferior dos valores de água aplicada.

No cálculo de $\overline{\mathrm{X}}_{\mathrm{q}}$, empregou-se a seguinte equação:

$\overline{\mathrm{X}}_{\mathrm{q}}=4\left[\int_{0}^{1} \hat{\mathrm{X}} \mathrm{da}-\int_{0}^{3 / 4} \hat{\mathrm{X}} \mathrm{da}\right]$.

Se ao introduzir a definição de $\hat{X}$ (equação 2) na equação 19 e simplificando-a, obteve-se o seguinte:

$\bar{X}_{\mathrm{q}}=\mathrm{X}_{\min }+4\left(\mathrm{X}_{\max }-\mathrm{X}_{\min }\right)$

$\left[\int_{0}^{1}\left(1-\mathrm{a}^{\mathrm{n}}\right)^{\mathrm{m}} \mathrm{da}-\int_{0}^{3 / 4}\left(1-\mathrm{a}^{\mathrm{n}}\right)^{\mathrm{m}} \mathrm{da}\right]$

Substituindo a primeira e a segunda integral por suas respectivas soluções, equações 5 e 16, e considerando $\mathrm{a}_{\mathrm{L}}=0,75$, obteve-se o seguinte resultado:

$\bar{X}_{q}=X_{\text {min }}+4\left(X_{\text {max }}-X_{\text {min }}\right)\left[\frac{m}{(1+m n)} \frac{\Gamma(1 / n) \Gamma(m)}{\Gamma(1 / n+m)}\right]$

$\left\{1-\operatorname{DISTF}\left[\left(0,75^{-\mathrm{n}}-1\right) /(\mathrm{nm}+\mathrm{n}) ; 2(\mathrm{~m}+1) ; 2(1 / \mathrm{n})\right]\right\}$.

Exemplificando o uso da equação 21 com os dados da Tabela 1, utilizando a função DISTF[0,1511; 4,2566; 2,6264] $=0,9461$ da planilha Excel e os demais

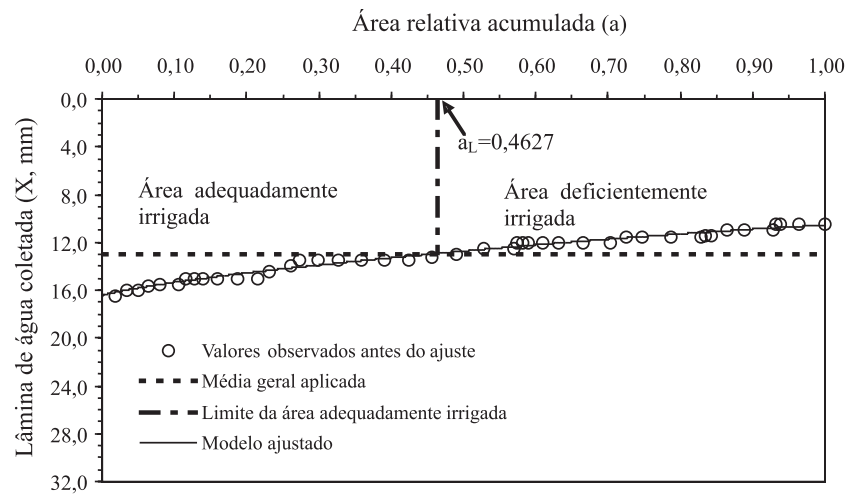

Figura 1. Distribuição dos valores das lâminas de água de um pivô central, medidas e ajustadas, tendo como parâmetros do modelo os seguintes valores: $\mathrm{n}=0,7615 ; \mathrm{m}=1,1283$; $X_{\max }=16,5 \mathrm{~mm} ; X_{\min }=10,6 \mathrm{~mm}$, para uma média aplicada, $\bar{X}=12,96 \mathrm{~mm}$. 
parâmetros já determinados, obteve-se a média do quartil inferior $\overline{\mathrm{X}}_{\mathrm{q}}=11,11 \mathrm{~mm}$. Com este resultado, considerando a média $\bar{X}=12,96 \mathrm{~mm}$ já conhecida e empregando a definição expressa pela equação 18 , obteve-se o valor de CUD $=85,73 \%$. O valor de CUD, calculado diretamente com os dados medidos (Tabela 1), foi de $85,72 \%$, indicando que o modelo reproduziu bem o caso avaliado.

Assumindo que a lâmina média aplicada (Figura 1) é igual à lâmina de água requerida pela cultura, as frações de lâmina que superam a média devem ser consideradas excedentes. A área correspondente à integral do perfil de água aplicada $\hat{X}$ (equação 2) em relação à variável "a" no intervalo de 0 a 1 - cujo resultado numérico é o mesmo que o da lâmina média aplicada $(\overline{\mathrm{X}})$ obtida pela equação 6 - representa o volume total de água aplicada, $\mathrm{V}_{\mathrm{a}}$, expresso em termos de lâmina de água por unidade de área. Assim, para o caso do pivôcentral analisado, $\mathrm{V}_{\mathrm{a}}=\overline{\mathrm{X}}=12,96 \mathrm{~mm}$.

Os valores de lâmina excedentes, quando integradas no domínio da variável "a" no intervalo de $0 \mathrm{a} \mathrm{a}_{\mathrm{L}}$, geram o volume de água excedente $\left(\mathrm{V}_{\mathrm{e}}\right)$. A diferença entre o volume de água aplicado $\left(\mathrm{V}_{\mathrm{a}}\right)$ e o excedente $\left(\mathrm{V}_{\mathrm{e}}\right)$ resulta no volume de água útil $\left(\mathrm{V}_{\mathrm{u}}\right)$. No cálculo de $\mathrm{V}_{\mathrm{u}}$, foi necessário, primeiramente, determinar $\mathrm{V}_{\mathrm{e}}$ e, então, utilizar a definição $\mathrm{V}_{\mathrm{u}}=\mathrm{V}_{\mathrm{a}}-\mathrm{V}_{\mathrm{e}}$. Por sua vez, considerando que o processo de otimização da equação 1 resulta na divisão igualitária das áreas em excesso e em deficiência, deduziu-se que $\mathrm{V}_{\mathrm{e}}=0,5|\overline{\mathrm{D}}|$, desde que a lâmina média aplicada seja igual à lâmina de água necessária. Assim, no exemplo analisado (Tabela 1 e Figura 1), em que $|\overline{\mathrm{D}}|=1,38 \mathrm{~mm}$, então $\mathrm{V}_{\mathrm{e}}=0,69 \mathrm{~mm}$ e, por conseguinte, $V_{u}=12,27 \mathrm{~mm}$.

Com as variáveis $\mathrm{V}_{\mathrm{u}}$ e $\mathrm{V}_{\mathrm{a}}$ definidas, a eficiência de aplicação $\left(\mathrm{E}_{\mathrm{a}}\right)$ da irrigação pode ser expressa por:

$\mathrm{E}_{\mathrm{a}}=100\left(\mathrm{~V}_{\mathrm{u}} / \mathrm{V}_{\mathrm{a}}\right)$

em que $\mathrm{E}_{\mathrm{a}}$ é dada em porcentagem. Desse modo, no caso estudado (Figura 1), a eficiência de aplicação resultou em $E_{a}=94,68 \%$, correspondendo a uma perda por percolação de 5,32\%. O cálculo desse valor, utilizando diretamente os dados medidos envolve aproximações gráficas e numéricas do perfil de distribuição, o que introduz imprecisões na análise.

Destaca-se que os indicadores de uniformidade (CUC ou CUD) servem apenas como guia no julgamento do desempenho do sistema de irrigação em relação a sua habilidade em distribuir espacialmente a água na área irrigada. Do ponto de vista do manejo, é necessário estabelecer a meta em termos de área que se deseja manter adequadamente irrigada. Sistemas com baixa uniformidade, normalmente, resultam em índices de desempenho menores, requerendo, portanto, maiores lâminas no ajuste da quantidade de água requerida pela cultura. Assim, a área adequadamente irrigada é ampliada. No entanto, a eficiência de aplicação de água é reduzida. O ajuste da área adequadamente irrigada é uma operação na qual se busca o ponto ótimo entre o porcentual de área que deve receber aplicações de água em quantidades iguais ou superiores à lâmina requerida pela cultura para satisfazer exigências de produtividade, com o menor porcentual de excesso possível. A água aplicada em excesso representa aumento no consumo de energia, reduzindo a margem de lucro do produtor, com possíveis impactos sobre a lixiviação de nutrientes e o meio ambiente.

Uma vez estabelecido o porcentual de área que se deseja irrigar adequadamente, a lâmina média a ser aplicada deverá ser ajustada de modo que o perfil de distribuição de água resultante intercepte a lâmina de água requerida no ponto correspondente ao valor preestabelecido de área adequadamente irrigada. $\mathrm{Na}$ Figura 2, apresentam-se as distribuições dos valores de água correspondentes ao ajuste do porcentual de área adequadamente irrigada de 46,27\% para $90,00 \%$. A linha horizontal tracejada representativa da média de água aplicada correspondente ao primeiro perfil, a qual é tida como a lâmina requerida pelo cultivo, permanece

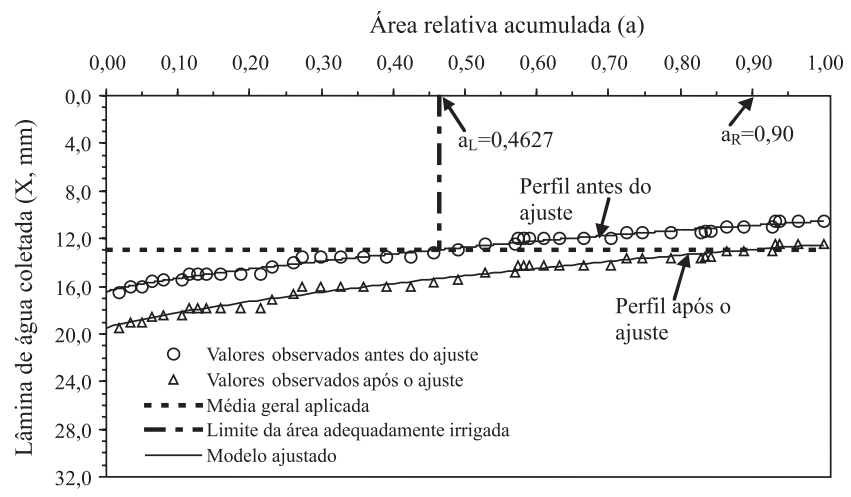

Figura 2. Distribuição dos valores de água aplicada por um pivô central, ordenados de forma decrescente em função da área irrigada, expressa em termos da área relativa acumulada, indicando dois resultados distintos de área adequadamente irrigada $(46,27 \%$ e $90,00 \%)$. 
estacionária, enquanto o segundo perfil deve ser deslocado para uma posição correspondente à meta de $90,00 \%$ de área adequadamente irrigada. No caso desse ajuste, haverá aumento nos volumes de água aplicada e excedente, resultando em decréscimo da eficiência de aplicação.

Tomando por base a assertiva de que o formato do perfil de distribuição dos valores de lâmina de água aplicada não se altera com a variação da lâmina média, o ajuste da área adequadamente irrigada pode ser alcançado, simplesmente, pelo deslocamento de toda a distribuição, para baixo ou para cima, conforme o desejado. Esse deslocamento pode ser definido por um fator de proporcionalidade, denominado Fator de Adequação da Irrigação, representado por $\mathrm{F}_{\mathrm{AI}}$ e expresso, matematicamente, da seguinte forma:

$\mathrm{F}_{\mathrm{AI}}=\hat{\mathrm{X}}_{\mathrm{R}} / \hat{\mathrm{X}}$

em que $\hat{\mathrm{X}}_{\mathrm{R}}$ é o novo valor de lâmina ou volume de água, correspondente a um dada área relativa acumulada (a), após o deslocamento de toda a distribuição; e X́ representa os valores de lâmina ou volume da distribuição de água antes do ajuste (Figura 2).

Qualquer que seja $\hat{X}$, ao ser multiplicado pelo Fator de Adequação da Irrigação $\left(\mathrm{F}_{\mathrm{AI}}\right)$, seu valor é transformado no $\hat{X}_{R}$ correspondente ao mesmo valor de área relativa acumulada. Seguindo essa lógica, foram estabelecidas as seguintes igualdades:

$\hat{\mathrm{X}}_{\mathrm{R}}=\left(\mathrm{F}_{\mathrm{AI}}\right) \hat{\mathrm{X}} ; \mathrm{X}_{\mathrm{R}_{\min }}=\left(\mathrm{F}_{\mathrm{AI}}\right) \mathrm{X}_{\text {min }}$

$\mathrm{X}_{\mathrm{R}_{\max }}=\left(\mathrm{F}_{\mathrm{AI}}\right) \mathrm{X}_{\text {max }} ; \overline{\mathrm{X}}_{\mathrm{R}}=\left(\mathrm{F}_{\mathrm{AI}}\right) \overline{\mathrm{X}}$

em que $X_{R_{\min }}$ e $X_{R_{\max }}$ são, respectivamente, os valores mínimos e máximos das lâminas de água ou vazões da distribuição deslocada; e $\overline{\mathrm{X}}_{\mathrm{R}}$ representa a média das lâminas ou vazões aplicadas com a nova distribuição.

Observando-se a Figura 2, verifica-se que a interseção da linha representativa da quantidade média original de água aplicada $\bar{X}$ com o perfil descrito por $\hat{X}_{R}$ ocorre em $a_{R}$, de modo que, utilizando a definição proposta na equação 2, deduziu-se a seguinte expressão:

$\bar{X}=X_{R_{\min }}+\left(X_{R_{\max }}-X_{R_{\min }}\right)\left(1-a_{R}^{n}\right)^{m}$.

Desse modo, introduzindo as relações da equação 24 na equação 25 , obteve-se a equação 26 :

$\bar{X}=F_{A I}\left[X_{\text {min }}+\left(X_{\text {max }}-X_{\text {min }}\right)\left(1-a_{R}^{n}\right)^{m}\right]$,

resultando no fator de adequação proposto:
$\mathrm{F}_{\mathrm{AI}}=\frac{\overline{\mathrm{X}}}{\left[\mathrm{X}_{\text {min }}+\left(\mathrm{X}_{\text {max }}-\mathrm{X}_{\text {min }}\right)\left(1-\mathrm{a}_{\mathrm{R}}^{\mathrm{n}}\right)^{\mathrm{m}}\right]}$

em que $\frac{\bar{X}}{X_{\max }} \leq F_{A I} \leq \frac{\bar{X}}{X_{\min }} \Leftrightarrow 0 \leq a_{R} \leq 1$.

Com os resultados do exemplo da Tabela 1 e considerando a meta preestabelecida de 90,00\% de área adequadamente irrigada, obteve-se o valor de $\mathrm{F}_{\mathrm{AI}}=1,1860$. Isso significa que para atingir $90,00 \%$ da área com irrigação adequada é necessário majorar o requerimento líquido de água em 18,60\% nesse sistema de irrigação.

Quanto ao desempenho da irrigação, o ajuste no perfil de distribuição de água altera somente a eficiência de aplicação de água, mantendo inalterados os indicadores de uniformidade. No cálculo da eficiência de aplicação resultante $\mathrm{E}_{\mathrm{a}_{\mathrm{R}}}$, foi necessário determinar as quantidades de água ajustadas: volume total aplicado $\mathrm{V}_{\mathrm{a}_{\mathrm{R}}}$, volume útil $\mathrm{V}_{\mathrm{u}_{R}}$ e volume excedente $\mathrm{V}_{\mathrm{e}_{\mathrm{R}}}$. $\mathrm{O}$ volume total de água aplicado $\mathrm{V}_{\mathrm{a}_{\mathrm{R}}}$ foi obtido diretamente pela multiplicação de $\mathrm{F}_{\mathrm{AI}}$ por $\overline{\mathrm{X}}$, que, no caso exemplificado, resultou em $\mathrm{V}_{\mathrm{a}_{\mathrm{R}}}=15,37 \mathrm{~mm}$.

Para o cálculo do volume excedente após o ajuste do perfil de água aplicada, utilizou-se a seguinte definição:

$\mathrm{V}_{\mathrm{e}_{\mathrm{R}}}=\int_{0}^{\mathrm{a}_{\mathrm{R}}}\left(\hat{\mathrm{X}}_{\mathrm{R}}-\overline{\mathrm{X}}\right) \mathrm{da}$.

Substituindo $\hat{X}_{R}$ por $\left(\mathrm{F}_{\mathrm{AI}}\right) \hat{\mathrm{X}}$ (equação 24) e efetuando-se as devidas simplificações, obteve-se o seguinte resultado:

$\mathrm{V}_{\mathrm{e}_{\mathrm{R}}}=\mathrm{F}_{\mathrm{AI}}\left[\mathrm{X}_{\min } \mathrm{a}_{\mathrm{R}}+\left(\mathrm{X}_{\max }-\mathrm{X}_{\min }\right)_{0}^{\mathrm{a}_{\mathrm{R}}}\left(1-\mathrm{a}^{\mathrm{n}}\right)^{\mathrm{m}} \mathrm{da}\right]-\overline{\mathrm{X}} \mathrm{a}_{\mathrm{R}}$.

Substituindo $a_{L}$ por $a_{R}$ na equação 16 , obteve-se a seguinte solução para $\mathrm{V}_{\mathrm{e}_{\mathrm{R}}}$ (equação 29):

$\mathrm{V}_{\mathrm{e}_{\mathrm{R}}}=\mathrm{F}_{\mathrm{AI}}\left\{\mathrm{X}_{\text {min }} \mathrm{a}_{\mathrm{R}}+\left(\mathrm{X}_{\max }-\mathrm{X}_{\text {min }}\right) \operatorname{DISTF}\left[\frac{\left(\mathrm{a}_{\mathrm{R}}^{-\mathrm{n}}-1\right)}{(\mathrm{nm}+\mathrm{n})} ;\right.\right.$

$\left.2(\mathrm{~m}+1) ; 2(1 / \mathrm{n})]\left[\frac{\mathrm{m}}{(1+\mathrm{mn})} \frac{\Gamma(1 / \mathrm{n}) \Gamma(\mathrm{m})}{\Gamma(1 / \mathrm{n}+\mathrm{m})}\right]\right\}-\overline{\mathrm{X}} \cdot \mathrm{a}_{\mathrm{R}}$.

Assim, para os dados do exemplo utilizado na Tabela 1, considerando os seguintes parâmetros: $\mathrm{F}_{\mathrm{AI}}=1,1860$; $\mathrm{a}_{\mathrm{R}}=0,9 ; \mathrm{n}=0,7615 ; \mathrm{m}=1,1283 ; \mathrm{X}_{\max }=16,5 \mathrm{~mm}$; $\mathrm{X}_{\text {min }}=10,6 \mathrm{~mm}$, determinou-se $\mathrm{V}_{\mathrm{e}_{\mathrm{R}}}=2,43 \mathrm{~mm}$, em que DISTF[0,0515; 4,2566; 2,6264] $=0,9913$. Neste caso, o excesso de água aplicada foi de $15,81 \%$ em relação ao total $\left(\mathrm{V}_{\mathrm{a}_{\mathrm{R}}}=15,37 \mathrm{~mm}\right)$. Desse modo, o volume útil ajustado foi igual a $\mathrm{V}_{\mathrm{u}_{\mathrm{R}}}=12,94 \mathrm{~mm}$, resultando em uma efi- 
ciência de aplicação $\mathrm{E}_{\mathrm{a}_{\mathrm{R}}}=84,19 \%$. Nessa aplicação (Tabela 1), concluiu-se que com a majoração da lâmina em $18,60 \%$, a área adequadamente irrigada passou de $46,27 \%$ para $90,00 \%$, reduzindo a eficiência de aplicação de $94,68 \%$ para $84,19 \%$ e aumentando o volume excedente de água de 5,32\% para 15,81\%.

\section{Conclusões}

1. O modelo desenvolvido é apropriado para descrever a distribuição dos valores de lâmina de água aplicada e fornece os parâmetros necessários para a avaliação de desempenho de sistemas de irrigação.

2. O fator de adequação da irrigação $\left(\mathrm{F}_{\mathrm{AI}}\right)$ desenvolvido, ao englobar os indicadores de desempenho da irrigação, simplifica os procedimentos de análise e fornece uma ferramenta direta para o cálculo da lâmina de água requerida para irrigação.

\section{Agradecimentos}

Ao CNPq, pela concessão de bolsa PROFIX ao quarto autor; aos colegas da Embrapa Cerrados que contribuíram com sugestões para melhoria deste trabalho.

\section{Referências}

ABRAMOWITZ, M.; STEGUN, I.A. Handbook of mathematical functions with formulas, graphs, and mathematical tables. Washington, D.C.: U.S. Government Printing Office, 1972.
CHAUDHRY, F.H. Nonuniform sprinkler irrigation application efficiency. Journal of the Irrigation and Drainage Division, v.104, p.165-178, 1978.

ELLIOT, R.L.; HART, W.E.; LOFTS, J.C.; NELSON, J.D. Comparison of sprinkler uniformity models. Journal of the Irrigation and Drainage Division, v.106, p.321-330, 1980.

GENUCHTEN, M.T. van. A closed-form equation for predicting the hydraulic conductivity of unsaturated soils. Soil Science Society of America Journal, v.44, p.892-898, 1980.

HAHN, R.H; ROSENTRETER, E.E. (Ed.). ASAE Standards 1989: Standards, engineering practices and data developed and adopted by the American Society of Agricultural Engineers. 36th ed. St. Joseph: ASAE, 1989. 659p.

HART, W.E.; PERI, G.; NORUM, D.I. Optimal seasonal irrigation application analysis. Journal of the Irrigation and Drainage Division, v.106, p.221-235, 1980.

HART, W.E.; REYNOLDS, W.N. Analytical design of sprinkler systems. Transactions of the American Society of Agricultural Engineers, v.8, p.83-89, 1965.

KARMELI, D. Distribution patterns and losses for furrow irrigation. Journal of the Irrigation and Drainage Division, v.104, p.59-68, 1978.

SILVA, E.M. da; HART, W.E. Modelo estatístico de distribuição de água infiltrada aplicado à irrigação deficiente. In: CONGRESSO NACIONAL DE IRRIGAÇÃO E DRENAGEM, 9., 1992, Fortaleza. Anais. Fortaleza: ABID, 1992. v.1, p.469-492.

WARRICK, A.W. Interrelationships of irrigation uniformity terms. Journal of the Irrigation and Drainage Engineering, v.109, p.317-332, 1983.

Recebido em 19 de fevereiro de 2004 e aprovado em 4 de maio de 2004

Pesq. agropec. bras., Brasília, v.39, n.8, p.741-748, ago. 2004 